\title{
Self-Tuning VGPI Controller Based on ACO Method Applied for WTGS System
}

\author{
M. Madaci, D. Kerdoun \\ LGEC-Research Laboratory, Department of Electrical Engineering, Constantine 1 University, 25000 Constantine, \\ Algeria
}

\begin{tabular}{l} 
Article Info \\
\hline Article history: \\
Received Mar 21, 2017 \\
Revised May 16, 2017 \\
Accepted May 21, 2017 \\
\hline
\end{tabular}

Keyword:

Ant colony optimization Algorithm (ACO)

PWM rectifier

Scheduling regulation

Variable gain PI (VGPI)

Wind turbine generation system

\begin{abstract}
The stability and the fast response are two parameters to evaluate the efficiency of any system, and the acknowledgement of the mathematic model studied and its parameters are strongly required. In order to build the regulation and the control of the system, different methods are used. Some are traditional (PI, PD, PID); whereas, others are modern (Fuzzy logic, neural networks, statistical algorithms, genetic algorithms, VGPI and so on). In this paper, we focused on the presentation of a new method which we call the scheduling regulation based on a particle swarm optimization. A stochastic diffusion search method that takes inspiration from the social behaviors of real ants with their environment. Ant colony optimization algorithms (ACO) presents a promising performance which is a selforganized regulation system with no need to the acknowledgment of both the mathematic model and the parameters of the systems from a side, and it can insure the stability and the fast response of the system from another side.
\end{abstract}

Copyright $@ 2017$ Institute of Advanced Engineering and Science. All rights reserved.

\section{Corresponding Author:}

Madaci Mansour,

Department of Electrical Engineering,

National Constantine University,

Road Ain Elbay, Constantine 25000, Algeria.

Email: zoldaioque@gmail.com

\section{INTRODUCTION}

In the last years, researchers have drawn attention to the intelligent algorithms that can give the high performance and treat the complex systems which are based on swarm intelligence. This latter is a new approach to solve stability problems that takes its inspiration from the real behavior of social insects and their interactions with the environment such as bees and ants. The SI also includes bird flocking, animal herding, bacterial growth and fish schooling. Existing swarm intelligence algorithms are Altruism algorithms, Ant colony optimization, Artificial Immune Systems, Charged System Search, Cuckoo search, Gravitational search algorithm, Intelligent Water Drops[1-2].

Ants always find the shortest path that links food to their nest after a long search without using a visual connection in the transmission of the information.

This kind of insects use the pheromone trails. Pheromones are chemicals capable of acting outside the body to create a link between each individual member of the colony [1] . They are secreted or excreted chemical factors that trigger a social response in members of the same species.

While any member of ant colony moves, it drops a certain pheromone on the floor which can be followed by other members of the same colony. The more ants follow this pheromone trails, the more attractive trails become to be followed by the other ants in the future [3].

The first ACO was proposed by Marco Dorigo in 1992[2]. It has a strong robustness and a good distributed calculative mechanism. It is easy to be combined with other methods like genetic algorithm [4-5]; computation, based on quantum mechanics, and continuous quantum ant colony optimization (CQACO) that integrates quantum computation into ACO [6]. Ant colony optimization focuses on performance of solving 
complex combinatorial optimization problems such as the aircraft problem [7], the DNA design [8], electronic and electrical domains [9] [6] [10].Many modified versions and new models of algorithms have been Proposed in different works to accelerate the decision process for the determination of optimal solution in path finding [11-15].

In this paper, we aim to give a new way for applying the artificial intelligence, the self-learning systems to be integrated in the control and the regulation systems of the wind renewable energy systems. This latter was considered as a major problem on the world energy from one part, and an effort to integrate renewable energy in the world's power system from another part by avoiding their system from the complex control.

\section{THE BASIC PRINCIPAL AND MATHEMATICAL MODEL OF ANT COLONY ALGORITHM}

Explaining research chronological, including research design, research procedure (in the form of algorithms, Pseudocode or other), how to test and data acquisition [1-3]. The description of the course of research should be supported references, so the explanation can be accepted scientifically [2], [4].

Tables and Figures are presented center, as shown below and cited in the manuscript.

The survey shows that ACO was used firstly in the Travelling Salesman Problem (TSP) as well as in other fields in various applications such as Job Scheduling problems and Telecommunication Network as an optimizer, Vehicle Routing; but it has never used in the regulation and the control system before. Therefore, we aim, through this paper, to open a new gate for the uses of the artificial intelligent in the regulation domain since it became the subject of many researches.

In many ant species, ants move to and from a food source depositing on the ground a substance called pheromone. An ant encountering a previously laid trail can detect the dense of pheromone trail. Other ants perceive the presence of pheromone and tend to follow tracks where pheromone concentration is higher and reinforce the trail with their own pheromone. Through this mechanism, the ant colony collectively, at last, marks the shortest path which has the largest pheromone amount. Actually, such simple indirect communication way among ants embodies a kind of collective learning mechanism. Ants are able to transport food to their nest in a remarkably effective way [2], [16].

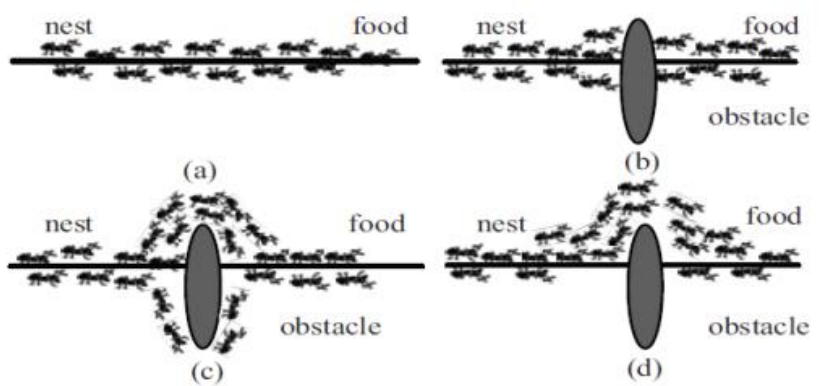

Figure 1. Self-adaptive Behavior of Ant Colony: (A) Real Ants Follow a Path Between Nest and Food

source; (B) An obstacle appears on the path: ants choose whether to turn left or right with equal probability; (C) Pheromoneis deposited more quickly on shorter path; (D) All ants have chosen shorter path [17]

The principle experiment of ACO shown in (Figure 2) is commonly known as "double bridge experiment", and it demonstrates the real behavior of the ants. First of all, we link an ant colony with a food source by two bridges. Starting by exploring the surroundings of the nest, the first ants randomly choose one of the two bridges depositing their pheromones along the path they take with random fluctuations. After a while, one of the bridges presents a higher concentration of pheromone than the other one, so it attracts more ants which bring a further amount of pheromone making the bridge more attractive [2]. As a result, the convergence of the whole colony is towards the use of the same path, and the ants avoid the obstacles on their way to the food source. 


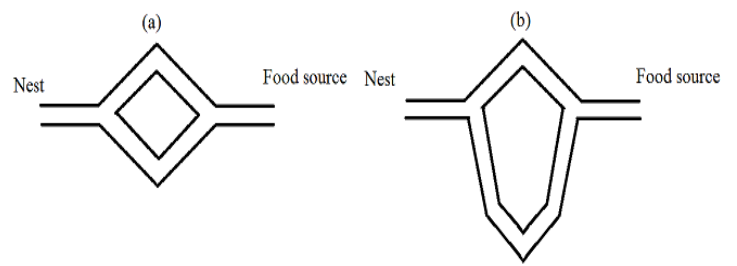

Figure 2. Experimental Setup for the Double Bridge Experiment [2]

The basic model of Ant Colony Optimization Algorithm is firstly given for the TSP problem of $n$ cities. We can define the parameters of basic ant colony algorithm as follow:

- $m$ will be the number of ants;

_ $\operatorname{dij}(i, j=1,2 \ldots n)$ will be the distance between city $i$ and city $j$.

_ $\tau i j(t)$ the residual pheromone along the distance linking the city $i$ and city $j$, at the moment $t, \tau i j(t=0)=0$.

Ants choose city to move to on the random proportional rule under the transition probability $P_{i j}^{k}$ from the starting point. In the moment $\mathrm{t}$, the transition probability for ant $\mathrm{k}$ in city $\mathrm{i}$ to select the city $\mathrm{j}$ is

$$
P_{i j}^{k}=\left\{\begin{array}{c}
\frac{\tau_{i j}^{\alpha}(t) \eta_{i j}^{\beta}(t)}{\sum_{j \in \text { allowedk }}\left[\tau_{i j}^{\alpha}(t) \eta_{i j}^{\beta}(t)\right]} \\
0
\end{array}\right.
$$

where, allowedk $=(0,1, \ldots \ldots, \mathrm{n}-1)$ is the city which ant $\mathrm{k}$ can choose at the next step, $\eta_{\mathrm{ij}}$ determines the expectations that ants go through from city $i$ to city $j, \alpha$ a constant that commands the relative influence of the pheromone trail , and $\beta$ a constant that determines the relative influence of the heuristic information.

In each time the ants complete the cycle the pheromone on each path is uploaded and adjusted according to:

$$
\begin{aligned}
& \tau_{i j}(t+n)=\rho \tau_{i j}(t)+\Delta \tau_{i j} \\
& \rho \in(0,1) \text { and } \Delta \tau_{i j}=\sum_{k=1}^{m} \Delta \tau_{i j}{ }^{k} \\
& \Delta \tau_{i j}{ }^{k}=\left\{\begin{array}{c}
\frac{Q}{L_{k}} \text { ant k goes through the path }(\mathrm{i}, \mathrm{j}) \text { in this cycle } \\
0
\end{array}\right.
\end{aligned}
$$

Since $\rho$ is pheromone evaporation rate and is related directly to the global searching ability and convergence rate of the algorithm, $\mathrm{Q}$ is the intensity coefficients of pheromone increased, Lk determines the length of the tour built by the k-th ant.

It's conventional that the ACO system is based on two feedbacks by ants. A positive feedback which is due to the deposit of pheromone that attracts other ants which will strengthen the paths, and a negative one which is due to the dissipation of the pheromone by evaporation that eliminates long paths and prevents the system from thrashing.

\section{SCHEDULING REGULATION BASED ON ANTS COLONYOPTIMIZATION USING RANDOM MATRIX NUMBER GENERATOR SOLUTION FOR THE WTGS}

Since our interest is to minimize the cost and to increase the performances of the WT generation system, we are going to use a SCIG (squirrel cage induction generator) machine because of its low cost as well as it needs rarely a maintenance instead of DFIG (doubly-fed induction generator) or the PMSG (permanent magnet synchronous generator) that need a lot of maintenance due to the brash system and the high cost respectively. Our method based on the self-organized regulator using ACO method is applied on 
the back to back system that step up the mechanical system which contains the WT and the SCIG. This latter has figured many strategies of control; however, those strategies are just theoretical methods.

\subsection{Power characteristics of wind turbines}

The captured power by wind turbines is:

$$
P_{\text {wind }}=\frac{\dot{m} v_{w}^{2}}{2}=\frac{\rho_{\text {wind }} A v_{w}^{3}}{2}
$$

The output power from the WT $P w$ is denoted by the following equation:

$$
P_{\text {wind }}=\frac{\rho_{\text {wind }} A v_{w}^{3} C_{p}(\lambda, \beta)}{2}
$$

Since $\mathrm{v}_{\mathrm{w}}$ is the wind speed, $\rho_{\text {wind }}$ is the air density, $A$ is the cross-section of rotor for WT, $C p$ is the power coefficient, $\beta$ is the pitch angle of blade, and $\lambda=\mathrm{Rw} / \mathrm{Vw}$ is the tip speed ratio that includes angular speed $\omega$ of rotor. The power extracted from the wind is thus proportional to the cube of the wind speed $\mathrm{v}$. The power coefficient $\mathrm{Cp}$, for a constant pitch angle $\beta$ depends only on the tip-speed ratio $\lambda$ which is defined as the ratio between the blade tip and the wind speeds as mentioned early. When the pitch angle remains constant, at low speeds, there exists a tip-speed ratio $\lambda$ ref for which the power coefficient curve $\mathrm{Cp}$ is optimal [18].

The WTG using a squirrel-cage induction generator with an output $P_{\text {generator }}$ can be expressed by:

$$
P_{\text {generator }}(w)=\frac{-3 V^{2} s(1+s) R_{2}}{\left(R_{2}-s R_{1}\right)+s^{2}\left(X_{1}+X_{2}\right)^{2}}
$$

where $V$ is phase voltage, the ratio: $s=\left(\omega_{0}-\omega\right) / \omega$ demonstrates the slip that includes synchronous angular speed $\omega_{o}$ of rotor, $R_{1}$ is stator resistance, $R_{2}$ is rotor resistance, $X_{1}$ is stator reactance, $X_{2}$ is rotor reactance. From above mentioned expression, $P g$ is function of $\omega$, Pwind is function of $\omega, V w$ and $\beta$.

$$
P_{\text {generator }}(w)=P_{\text {wind }}(w, V w, \beta)
$$

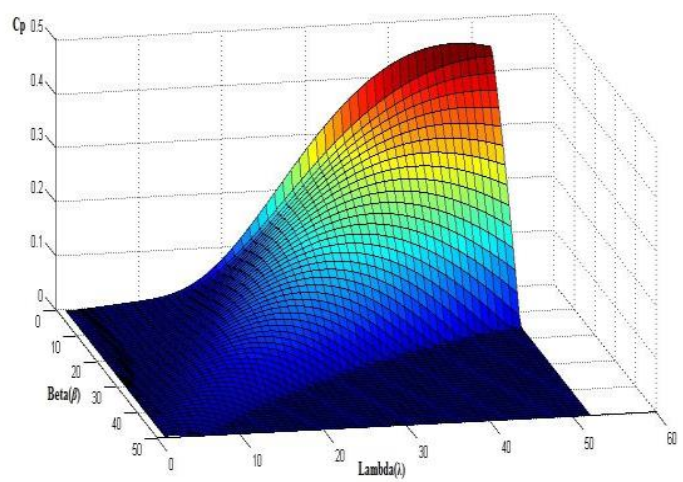

Figure. 3. Wind Turbine Characteristics Presentation

\subsection{Dynamical flexible model of WTGS}

The wind turbine system formed of a wind turbine, a SCIG and a power electronic converter shown in (Figure 5). The gearbox is used in order to increase the speed ratio from the low shaft coming from the WT to the high speed shaft going to the SCIG. Usually a single dominant model mode is used [19]. The most used model is the two mass model since this last one can give us the nearest approach to the real case [20]. 

written as:

The use of this model pushes to work on the following equation to describe our model that could be

$$
\left\{\begin{array}{c}
\ddot{\theta}_{m} \\
\ddot{\theta}_{t} \\
\dot{\theta}_{m} \\
\dot{\theta}_{t}
\end{array}\right\}=\left[\begin{array}{cccc}
\frac{v^{2} c}{J_{m}} & \frac{v c}{J_{m}} & \frac{-v^{2} k}{J_{m}} & \frac{v k}{J_{m}} \\
\frac{v c}{J_{t}} & \frac{-c}{J} & \frac{v k}{J_{t}} & \frac{-k}{J_{t}} \\
1 & 0 & 0 & 0 \\
0 & 1 & 0 & 0
\end{array}\right]\left\{\begin{array}{c}
\ddot{\theta}_{m} \\
\ddot{\theta}_{t} \\
\dot{\theta}_{m} \\
\dot{\theta}_{t}
\end{array}\right\}+\left\{\begin{array}{cc}
\frac{1}{J_{m}} & 0 \\
0 & \frac{1}{J_{m}} \\
0 & 0 \\
0 & 0
\end{array}\right\}\left\{\begin{array}{c}
T_{m} \\
T_{t}
\end{array}\right\}
$$

Where

Om : Orientation angle of the motor's shaft.

Ot : Orientation angle of turbine.

$\mathrm{V} \quad$ : Gear ratio of the multiplier.

C : Viscosity constant of the turbine's shaft.

$\mathrm{K}$ : Elasticity constant of the turbine's shaft.

$\mathrm{Jm} \quad$ : Inertia moment of the generator.

Jt : Inertia moment of the turbine.

Tm : Mechanical torque of the engine.

Ts : Mechanical torque of the turbine.

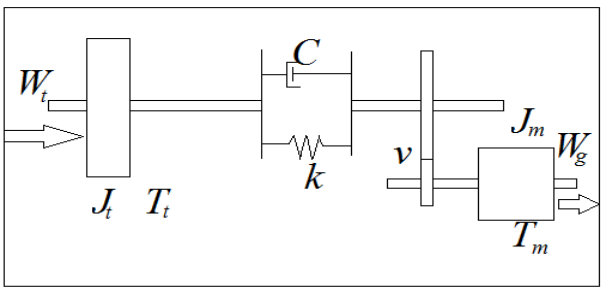

Figure 4. Wind Turbine with Flexible Transmission

\subsection{SCI Generator model in $(\alpha, \beta)$ Reference Frame}

In the WTG (wind turbine generation) systems we pay attention to the rotor speed and the load torque, only the SCIG model will be described, the SCIG model is presented considering the $(\alpha, \beta)$ as a frame of work. Many papers studied the machines model's for the WTG system on the same frame [21] according to this; the SCIG model will be as in [22].

$$
\left\{\begin{array}{c}
\frac{d}{d t}\left[\begin{array}{l}
I_{s} \\
\psi_{r}
\end{array}\right]=\left(\begin{array}{ll}
A_{11} & A_{12} \\
A_{21} & A_{22}
\end{array}\right)\left[\begin{array}{l}
I_{s} \\
\psi_{r}
\end{array}\right]+\left[\begin{array}{l}
B_{1} \\
B_{2}
\end{array}\right] U_{s} \\
I_{s}=C\left[\begin{array}{l}
I_{s} \\
\psi_{r}
\end{array}\right]
\end{array}\right.
$$

Since

$$
\begin{aligned}
& I_{s}(t)=\left[\begin{array}{l}
i_{s \alpha} \\
i_{s \beta}
\end{array}\right], U_{s}(t)=\left[\begin{array}{l}
U_{s \alpha} \\
U_{s \beta}
\end{array}\right], \psi_{s}(t)=\left[\begin{array}{l}
\psi_{s \alpha} \\
\psi_{s \beta}
\end{array}\right] \\
& A_{11}=\left(\begin{array}{cc}
-a & 0 \\
0 & -a
\end{array}\right), A_{12}=\left(\begin{array}{cc}
\frac{L_{m}}{b} & \frac{w L_{m}}{b} \\
\frac{-w L_{m}}{b} & \frac{L_{m}}{b}
\end{array}\right)
\end{aligned}
$$




$$
\begin{aligned}
& A_{21}=\left(\begin{array}{cc}
\frac{L_{m}}{T_{r}} & 0 \\
0 & \frac{L_{m}}{T_{r}}
\end{array}\right), A_{22}=\left(\begin{array}{cc}
\frac{-1}{T_{r}} & -w \\
w & \frac{-1}{T_{r}}
\end{array}\right) \\
& B_{1}=\left(\begin{array}{cc}
\frac{-1}{\sigma L_{s}} & 0 \\
0 & \frac{1}{\sigma L_{s}}
\end{array}\right), B_{2}=\left(\begin{array}{ll}
0 & 0 \\
0 & 0
\end{array}\right), a=\left(\frac{L_{r}^{2} R_{s}+L_{m}^{2} R_{r}}{\sigma L_{s} L_{r}^{2}}\right) \\
& C=\left(\begin{array}{llll}
1 & 0 & 0 & 0 \\
0 & 1 & 0 & 0
\end{array}\right), b=\sigma L_{s} L_{r}, \sigma=1-\frac{L_{m}^{2}}{L_{r} L_{s}}
\end{aligned}
$$

\subsection{Full Converter Model and Control}

In order to provide the magnetizing current for the reactive power, for the systems connected directly to the grid they draw it from the grid. However if the WTGS is in a stand-alone system, the SCIG should be connected directly to the grid through capacitor bank linked to the stator. Or a back to back converters which leads to use several configurations that allow us to work with different frequencies on each side [20].

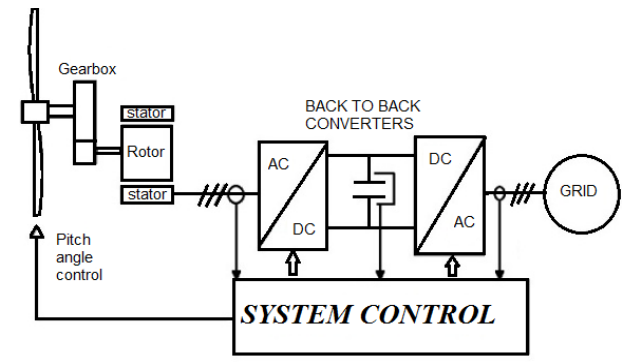

Figure 5. Structure of AC/DC/AC Power Electronic Converter System (Back to Back Converter) in Wind Power System

The model of the grid and the power electronic converter is usually given by the following equations:

$$
\begin{aligned}
& \left(V_{\text {kline }}-V_{\text {yline }}\right)=r \cdot i_{\text {kline }}+L \cdot \frac{d i_{\text {kline }}}{d t} \\
& \frac{d i_{\text {kline }}}{d t}=\frac{1}{L}\left[\left(V_{\text {kline }}-V_{\text {lline }}\right)-r \cdot i_{\text {kline }}\right]
\end{aligned}
$$
the converter.

Which $\mathrm{k}=1,2,3$ and $\gamma=1,2,3$ designing the three phases respectively of both the line and

The DC voltage control requires a feedback control loop, the DC bus voltage $\mathrm{V}_{\mathrm{dc}}$ is compared with a reference $V_{R E F}$, and the error generated "e" is used to generate a template waveform. This template will be used then to generate the command through a PWM command strategy.

To perform the regulation of the DC bus voltage, we open the way to many suggestions such as: the perfect conversion between DC /AC sides, the condenser voltage is almost constant and we can neglect the grid side inductance loss. Then we can come to DC bus regulation shown on diagram block. 


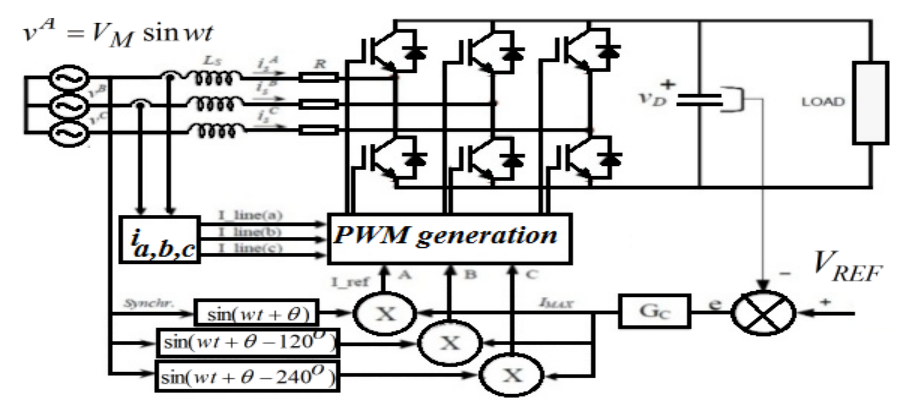

Figure 6. Structure of a Rectifier Based on a Current Loop Control
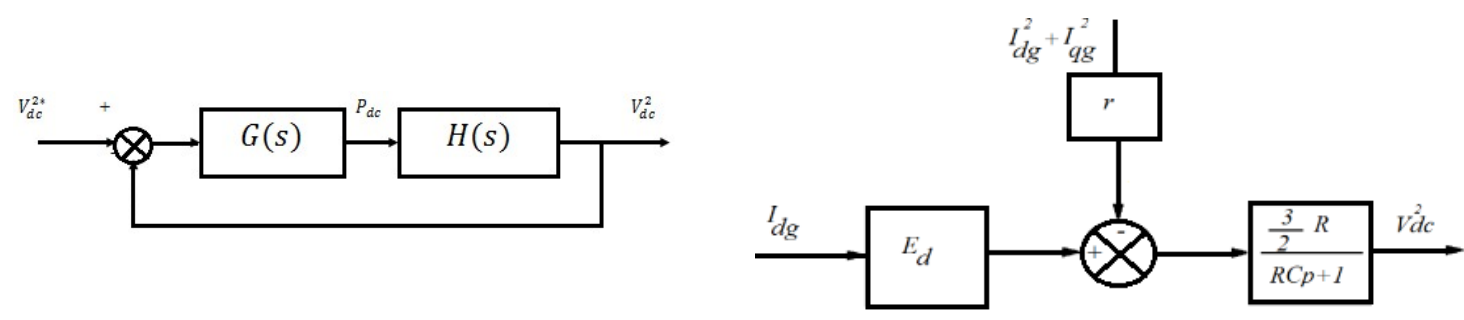

Figure 7. Diagram Block of DC Bus Regulation on a Closed Loop

Figure 8. Diagram Block for the DC Bus Regulation

Starting from the first suggestion that

$$
P_{d c} \cong P_{a c}
$$

$P=r \sum_{i=1}^{3} I_{g i}^{2}+V_{d c}+I_{d c}=\frac{3}{2} E_{d} I_{g d}$

Then

$P=\frac{3}{2} r\left(I_{g d}^{2}+I_{q d}^{2}\right)+V_{d c} I_{d c}$

$I_{d c}=C \frac{d V_{d c}}{d t}+\frac{V_{d c}}{R}$

$V_{d c} C \frac{d V_{d c}}{d t}+\frac{V_{d c}^{2}}{R}=\frac{3}{2} E_{d} I_{g d}-\frac{3}{2} r\left(I_{g d}^{2}+I_{q d}^{2}\right)$

$V_{d c}^{2}=\frac{\frac{3}{2} R E_{d} I_{g d}}{R C p+1}-\frac{\frac{3}{2} r\left(I_{g d}^{2}+I_{q d}^{2}\right)}{R C p+1}$

Since:

$\mathrm{V}_{\mathrm{dc}}$ is the DC voltage on the bus, and $\mathrm{I}_{\mathrm{dg}}, \mathrm{I}_{\mathrm{qg}}$ the direct and the quadrature components of the grid, $\mathrm{r}$ is the resistor of the link between the converter and the grid, $\mathrm{R}$ the resistor on the DC bus side 


\section{APPLICATION OF THE ACO IN THE DC BUS VOLTAGE REGULATION LOOP AND THE FIRST PROPOSED GENERATION OF SOLUTIONS IN THE ANT COLONY ALGORITHM}

The prediction of the solution generation rules are represented in the IFTHEN form and it can be described as: IF<conditions> THEN < generation>. In each iteration, the <condition> part usually contains a logic combination of predictor attributes, where each combination contains triple information of the crossed path <pheromone vector, path length vector, cross time vector>.

The suggestion of the solutions in the proposed method are assured through a generator module that gives the proposition of the different possible solutions in a matrix $H_{n}^{M}$ which contains a vector $X_{n}$ of $\{\mathrm{n}\}$ elements and indexed under $\mathrm{M}$ generation.

The proposed model suggests that each element of the matrix $H_{n}^{M}$ composed by an (M x n) item represent artificial ants which represent possible solutions of the DC bus voltage regulation problem.

We suppose that $X_{n}^{M}$ is the vector of artificial ants $\left\{A_{1}^{M}, A_{2}^{M}, \ldots . ., A_{n-1}^{M-1}, A_{n}^{M}\right\}$ which every ant determines a solution, the execution start from the first element of the vector $\mathrm{X}$ getting each element in each iteration to the (n) element of the vector, We have $X_{n}^{M}$, when $\mathrm{M}=0$ elements are randomly created, which give the initial generation of solutions.

The ants in the AC (ant colony) algorithm find the optimal path through the pheromone trail using the $\mathrm{X}$ vector items as solution. For the fitness of the solution and making sure of the convergence, when the result of some ants (elements) from the vector X gave a response, the generator try to make that almost the elements of $X_{n}^{M}\left\{A_{1}^{M}, A_{2}^{M}, \ldots . ., A_{n-1}^{M-1}, A_{n}^{M}\right\}$ are surely converge to the optimum solution by generating another generation of solutions depending on the previous one and the best solution from the previous elements of the vector $X_{n-1}^{M-1}$.

So, the distribution of the generated solutions is accorded to the best solution of the generated vector figure 9. As the vector elements in the vector $\mathrm{X}$ are finite, the optimum solution is finite too.

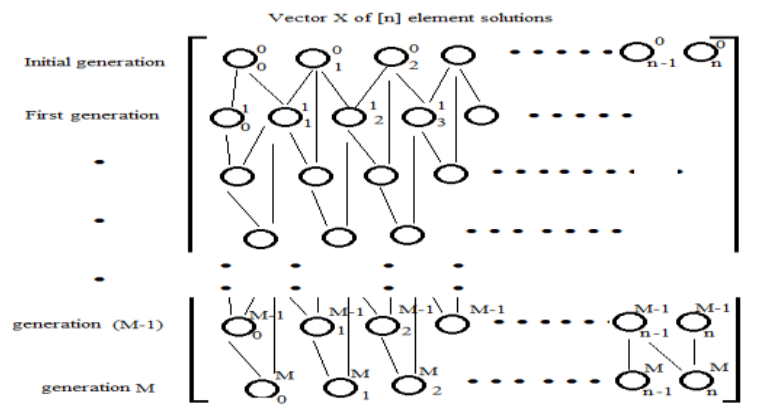

Figure 9. Solution Generation Process Used in the SG Module

The Generator module holds the generated Solutions under a vector that will be transferred to the main ACO system. Since the Generator works concurrently in parallel with the main system loop, the progress of system should be synchronized. For this reason, the solutions are transferred from the generator module through a shift register that contains in its turn the vector $X_{n}^{M}$.

The problem in the regulation system of the rectifier is digested during the transient state. The system tries to reach the given reference starting from the initial proposed solution.

\section{ALGORITHM REGULATION PROCESS USING ACO METHOD}

The following diagram demonstrates the regulation process using ant colony method. After the initialization, the system uploads the proposed solutions from the SG (Solution Generator) shift register module. Since every solution is represented by an individual ant, the internal cycle starts where the ants move according to the probability. The update of the pheromone and path states is done by the execution of the external cycle .The information translated to the SG module from the best solution shift register, when this last one creates a new generation of solutions according the previous ones. 


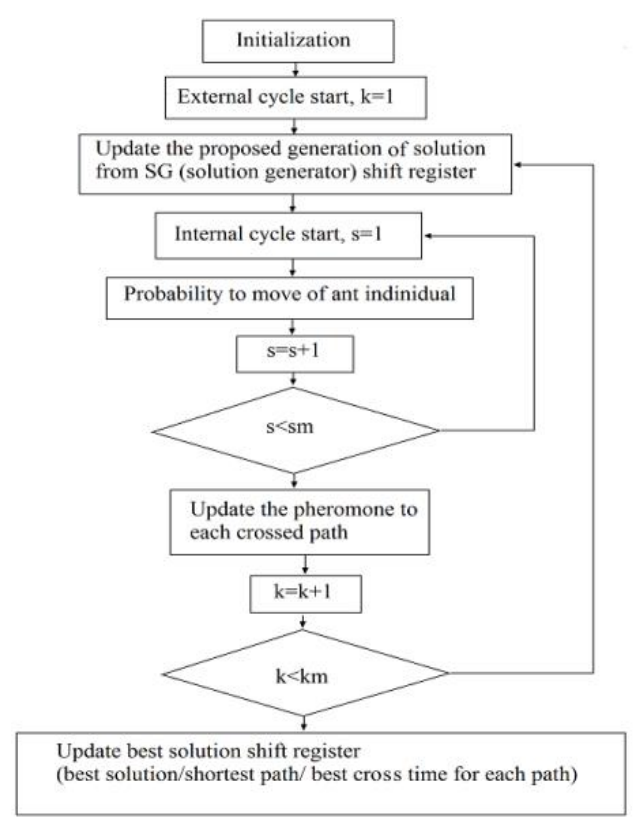

Figure 10. ACO Method Diagram with Internal and External Closed Loop

In order to increase the fitness of the solutions, to get a best convergence to the system and to minimize the execution time of the system, since the system in the last solution will keep just the best solutions that assure the convergence of the system, the idea is to create a single vector that combines between the best solutions, we applied a GRAPH SOLUTION DECOMPOSITION to the optimum solutions.

\section{GRAPH SOLUTION DECOMPOSITION}

The idea based on the decomposition of the graphs of each optimized solution, which represents the response of the system to reach the desired reference during the transient period to different parts or samples; the decomposition is done automatically according to:

1. The path length that takes the solution to reach the desired reference.

2. The time that takes the solutions to get through the path, since we inserted an auto-initialization counter that resets at the beginning of each new sample.

3. The first overtaking of the solution to the reference, which is a principle factor to determine the response of the system, this is related to a continuation factor" $\delta_{h}(n)$ ", which is the result of concurrently execution loop that measure the fluctuations and response of the system to the proposed solution. The core of the algorithm based on a close loop system for the decomposition determination of the graph rules.

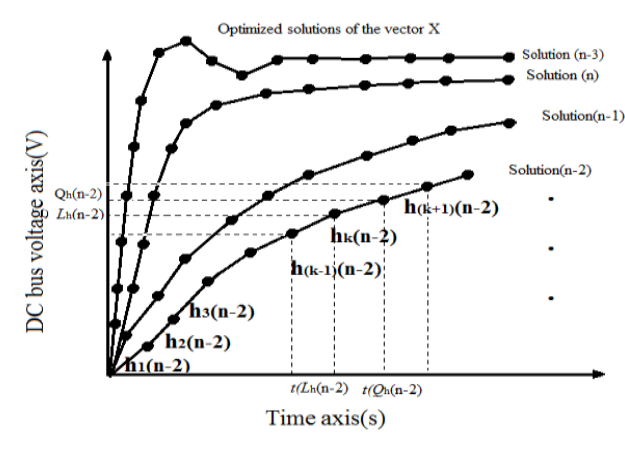

Figure 11. Graph Decomposition Topology with ACO Method 
First, the decomposition data is set under the form:

DecopositionSetTable(N;(PathLength,PathcrossedTime,Cont-inouationFactor" $\delta_{h}(n)$ ")), " $h$ " determine the number of the samples for each system response solution which determines the DC bus voltage curve for $(n)$ element of the vector $X_{n}^{M}$.

On this case $l_{h}^{n}$ and $Q_{h}^{n}$ will be respectively the MIN and the MAX of the sample $(h)$ from a part, from another part $t\left(Q_{h}(n)\right), t\left(l_{h}(n)\right)$ are the time accorded to the sample's extremities points $l_{h}^{n}$ and $Q_{h}^{n}$, this will be the output of the auto-initialized counter given under the form of vector $t_{h}(n)=\left\{\left(l_{h}(n)\right), t(l+1)_{h}(n), \ldots \ldots, t(Q-1)_{h}(n), t\left(Q_{h}(n)\right)\right\}$

In this case

$$
\delta_{h}(n)=\gamma_{h}(n)+\sigma_{h}(n)
$$

With

$$
\begin{aligned}
& \gamma_{h}(n)=\lambda /\left(t\left(Q_{h}(n)\right)-t\left(l_{h}(n)\right)\right) \\
& \sigma_{h}(n)=\left\{\begin{array}{l}
\sigma_{h-1}(n)+\lambda_{h}(n) \text { if } Q_{h}(n)>l_{h}(n) \\
\sigma_{h-1}(n)-\lambda_{h}(n) \text { if } Q_{h}(n)<l_{h}(n)
\end{array}\right.
\end{aligned}
$$

Since

$$
\lambda_{h}(n)=Q_{h}\left(t\left(Q_{h}(n)\right)\right)-l_{h}\left(t\left(l_{h}(n)\right)\right)
$$

The last finite optimized solution is given under two vectors which contain both: the best solution in each decomposition part $(h)$ of the $(n)$ optimized solutions, and the time required implicating these solutions, in parallel this constructs a schedule of successive solutions to the DC bus voltage regulation problem.

\section{SIMULATION RESULTS AND DISCUTION}

The simulation of the whole system was done using a co-simulation between two powerful platforms which are MATLAB and PSIM, the execution of the studied model and its control need powerful computation software and hardware. In order to achieve this task which allow us to reduce the simulation time from a part and minimize the errors from another we used the co-simulation between the both platforms by dividing the tasks between them.

Since MATLAB is among the most powerful calculation packages in the academia and industrial fields because of its advances capabilities in calculation, we are confided it with the mission of command and control part, the other part of the system that contains the power circuit was built on the electrical platform PSIM, since it's known with its fast execution and its precision in the power system cases.

The connection between both parts of the system the command and the power one was done using a SIMCOUPLER block. This creates a bridge between the two parts of the studied system.

The implementation of the command was divided on two parts, a part was built on an (m.file) which contains the principal parts of the command we cite the ant colony method, the solution generator closed loop module, and the drive of the execution cycles of the system.

The other part was built on SIMULINK which contains the different blocks of the connection and creates a link between the two parts of the whole system. 


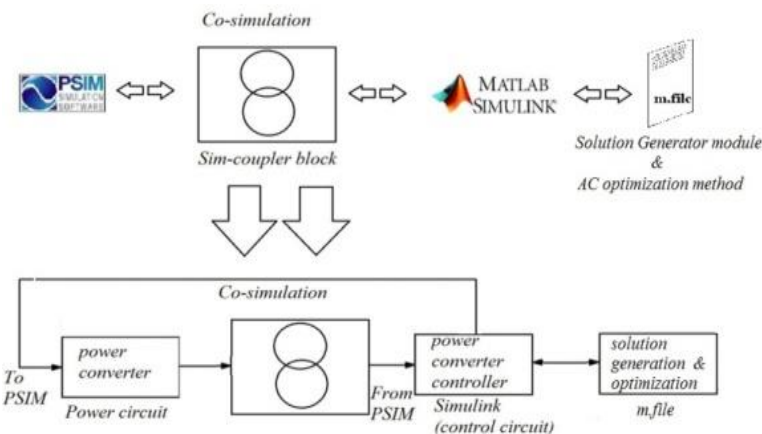

Figure 12. ACO Method Implementation Process Using Co-simulation MATLAB and PSIM Software

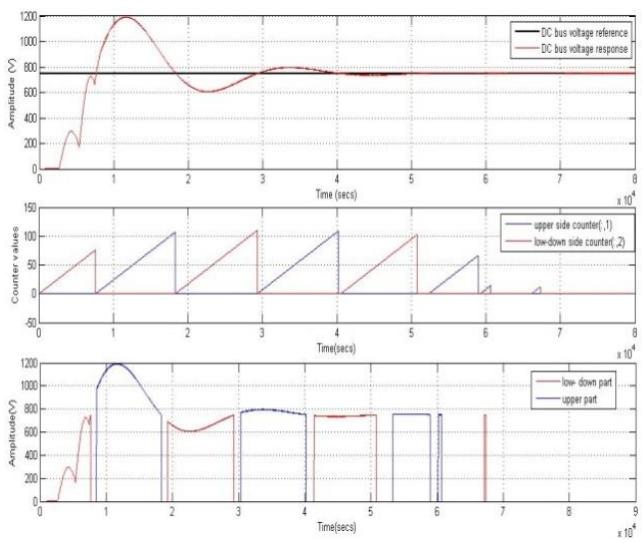

Figure 14. Auto-initialization Counters for the Overtaking Response Evaluation of the DC Bus Voltage

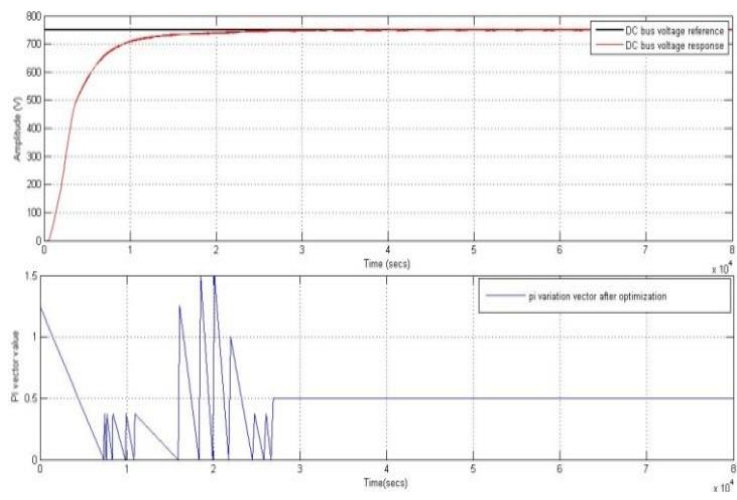

Figure 16. DC Bus Voltage Regulation Using the Combination of the Best Solutions Applied on a PreDeterminated Time Vector with a Pre-Determinated Solution Vector

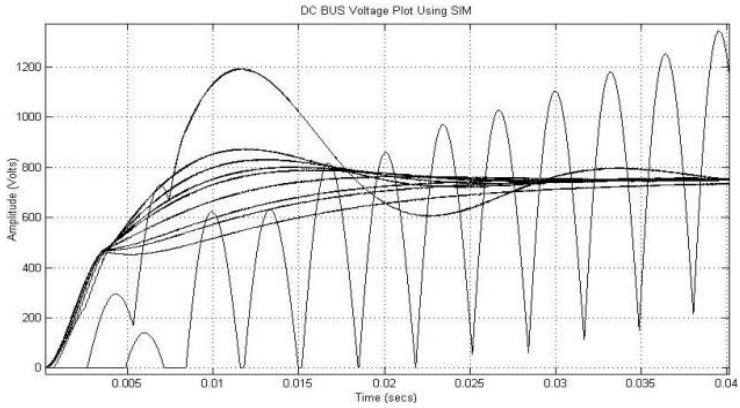

Figure 13. DC Bus Voltage Response for Best Ten Solutions Proposed by the SG Module of Ten Generations

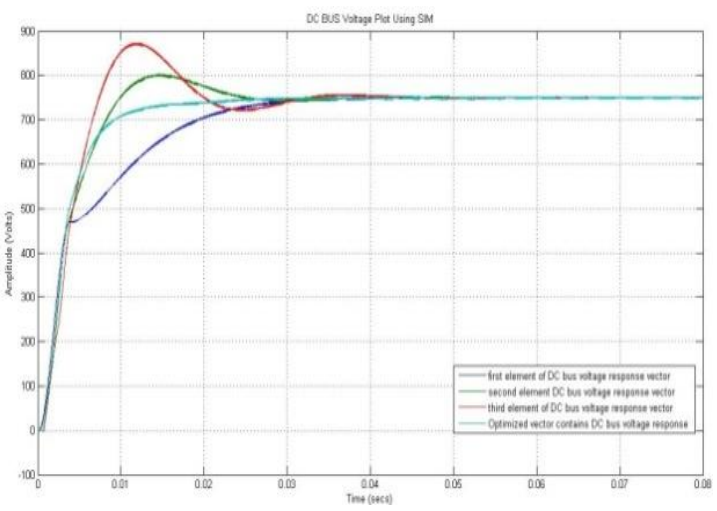

Figure 15. DC Bus Voltage Fitness Method Using the Graph Decomposition of the Best Three Solutions to Avoid the Overtaking. (The Fair Green Curven. Demonstrates the Last Optimized Solution from Graph Decomposition Method Using the Best Three Solutions Got from ACO Method)

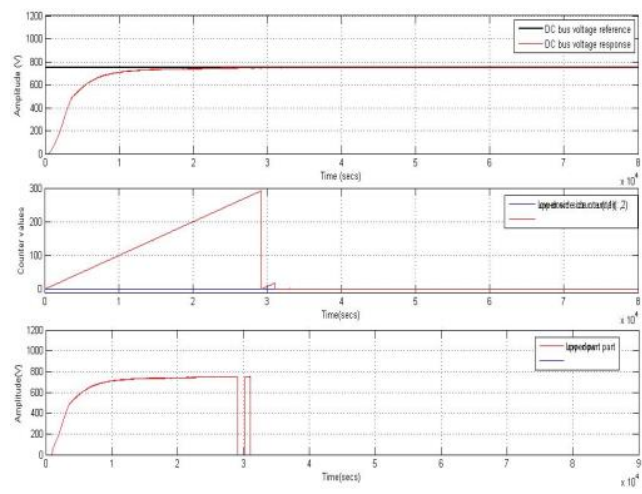

Figure 17. DC Bus Voltage Overtaking Response Evaluation of the Last Optimized Solution without Overtaking 


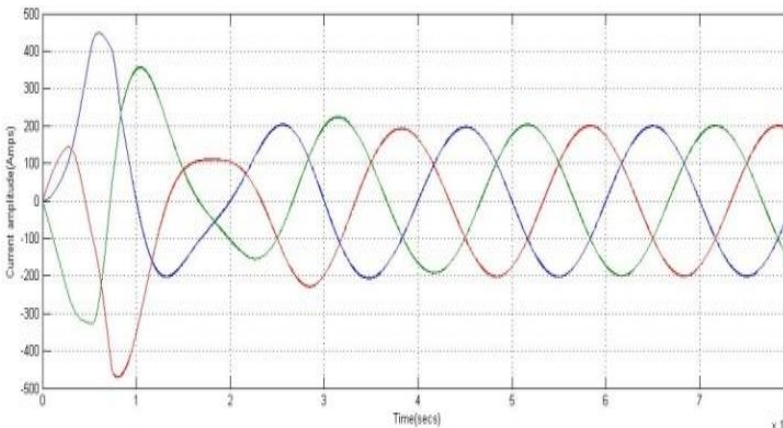

Figure 18. AC Side Currents Graph Using the Ordinary Regulation Based on a Standard PI

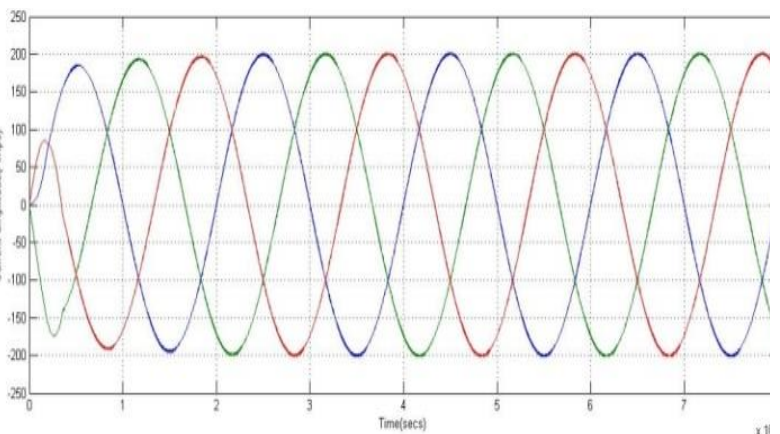

Figure 19. AC Side Currents Graph Using the Optimized Regulation Based on ACO Solution

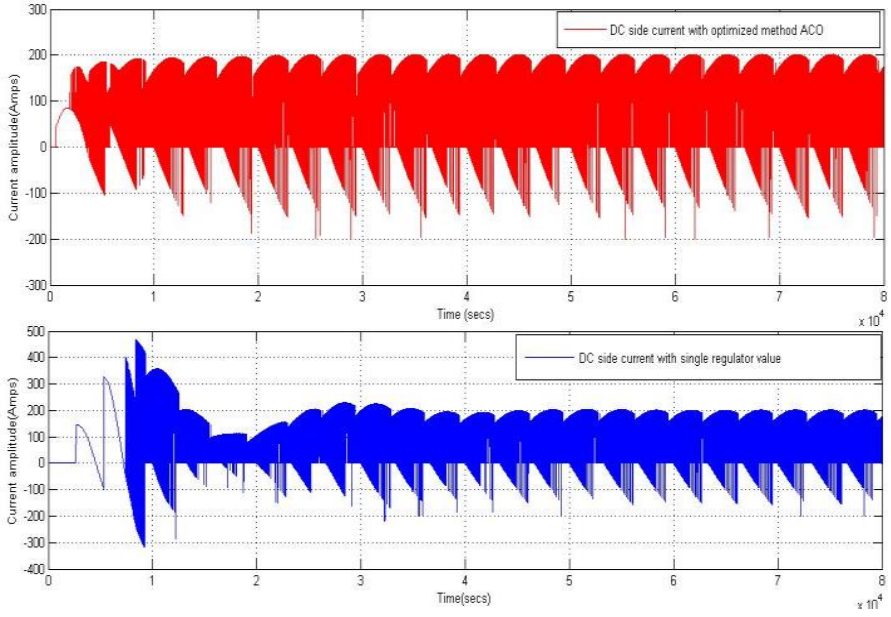

Figure. 20. DC Side Currents Graph Comparison Between the Optimized Solution and the Ordinary Regulation, (The Red Graph Determinates the DC Side Current with an ACO Regulation; Blue Graph Determinates the DC Side Current with a Standard Regulation)

\section{CONCLUSION}

In this paper, an ACO method reinforced with graph decomposition topology is presented, in order to create a new mode of regulation from a part, from another part to open the gates for a new domain based on artificial intelligent using the self-learning methods to be integrated in the electrical fields for the complicated systems and achieves the easy control of the nonlinear models to be as same as the linear ones.

The paper presents a study state of a WTGS containing a back to back converter system with a rectifier stepped up by an inverter. A PWM rectifier based on current control process was proposed, this type of devices is commonly used in many power systems, and during the last years it became the main subject of many studies looking to its important role especially in the renewable energy domains.

This kind of devices is classified as nonlinear system which is hard to control it and its behavior, since the output of the system and its parameters are depending on the parameters of the load on the output side and the power coming from the input side of this last one and strongly linked to the command applied on such systems.

The simulation of the whole system was done using a co-simulation between two powerful platforms, to create a powerful engine that can achieve the mission of simulation and control the model, in order to optimize the simulation time and the obtained results, the model was separated on two parts; the control part, and the electrical part, where the control part was built in MATLAB platform, looking to its precision in calculation, and the power electrical part which was built on PSIM platform since this last one is known with its fast execution in the power electrical domains. The ACO and the graph decomposition methods were built on an m.file which was used as a command unit for the whole system. 
The proposed method has satisfactorily assured the regulation and the control of the PWM rectifier system. In addition, this method allows the over-current protection during the transient state and the control of the load current

\section{ACKNOWLEDGEMENTS}

This material is based on work supported by the LGEC (laboratoire de génie élèctrique) laboratories, faculty of ELECTRICAL ENGINEERING. Constantine 1 University within the fund for research groups of electrical laboratories of the UNIVERSITY OF CONSTANTINE1 within the current research topic EnR 2015-2030 supported by the Ministry of Mines and Energy (MME) of Algeria.

\section{REFERENCES}

[1]. A.Tayade, L.Ragha, "Ant Colony Optimizer as an Adaptive Classifier,"International Conference on Communication, Information \& Computing Technology (ICCICT), pp. 1-6, 2012.

[2]. M. Dorigo, Marco Birattari and Thomas Stutzle, University Libre Bruxelles, Belgium, "Ant Colony Optimization, Artificial Ants as a Computational Intelligence Technique", IEEE Computational Intelligence Magazine, vol. 1, No.4, November 2006.

[3]. R.Seidova, J.Pozivil, "Implimentation of ant colony algorithms in Matlab," Institute of Chemical Technology, Department of Computing and Control Engineering, Technicka 5, Prague 6, 166 28, Crech Republic.

[4]. M. Yi, "The Research of Path-Oriented Test Data Generation Based on a Mixed Ant Colony System Algorithm and Genetic Algorithm,"8th International Conference on Wireless Communications, Networking and Mobile Computing (WiCOM), pp. $1-4,2012$.

[5]. Z. Meng, Q. Chen, "Hybrid genetic-ant colony algorithm based job scheduling method research of arc welding robot ," in Proc. IEEE International Conference on Information and Automation (ICIA), pp. 718 - 722, 2010.

[6]. W. Li, Q. Yin, X. Zhang, "Continuous quantum ant colony optimization and its application to optimization and analysis of induction motor structure," IEEE 5th International Conference on Bio-Inspired Computing: Theories and Applications (BIC-TA), pp. 313 - 317, 2010.

[7]. G. Xiao, B. Gu, Z. Jing, C. Yu, "An improved ant colony optimization approach for multi-aircraft horizontal escape maneuvers," 31st IEEE Conference in Digital Avionics Systems (DASC )AIAA, pp. 4D3-1 - 4D3-8, 2012.

[8]. Mustaza, S.M,.Abidin, A.F.Z,Ibrahim, Z, Shamsudin, M.A,Husain, A.R,Mukred, J.A.A, "A modified computational model of ant colony system in DNA sequence design,"IEEE Student Conference on Research and Development (SCOReD), pp. 169 - 173, 2011.

[9]. H. Liying, T. Hongmei, S. Zhijie, Z. Cunshan, Z. Hongdong, "Application of Improved Ant Colony Algorithm in Integrated Circuit Routing," 9th International Conference on Computer and Communications Security, ICCCS '09, pp. $44-47,2009$

[10]. L. Wei, X. Cui, "Study on Power Transformer Fault Test Sequence Optimization Based on Multi-colony Ant Colony Algorithm," International Conference on Intelligent Human-Machine Systems and Cybernetics (IHMSC), vol 2. pp. $127-130,2011$.

[11]. H.Liu, P.Li, Y.Wen, "Parallel Ant Colony Optimization Algorithm," in Proc. The Sixth World Congress on intelligent Control and Automation WCICA 2006, vol 1, pp.3222 - 3226, 2006.

[12]. Y.Shou, "A Bi-directional Ant colony algorithm for resource constrained project scheduling," in Proc.IEEE international Conference on Industrial Engineering and Engineering Management, IEEM, pp.1027-1031, 2007.

[13]. L. Liu, Y. Song, Y. Dai, "Cooperative multi-ant colony pseudo-parallel optimization algorithm," in Proc. IEEE International Conference on Information and Automation (ICIA), pp. 1269 - 1274, 2010.

[14]. C. Wu, "Ant colony multilevel path optimize tactic based on information consistence optimize," International Conference on Computer Application and System Modeling (ICCASM), vol 1, pp.V1-533 - V1-536,2010.

[15]. W.Junfang, N.Dongxiao, "Short-Term Power Load Forecasting Using Least Squares Support Vector Machines(LSSVM)," in Proc. Second International Workshop on Computer Science and Engineering, WCSE '09, vol 1, pp. $246-250,2009$.

[16]. R.Sakamoto, T.Senjyu, N.Urasaki, T.Funabashi, H.Fujita, H.Sekine, "Output Power Leveling of Wind Turbine Generators Using Pitch Angle Controlfor All Operating Regions in Wind Farm, " 2005 ISAP,2005.

[17]. J.Y.Dieulot, L.Chalal, F.Colas,"A vibration damping MPPT strategy for wind turbines using delayed feedback," in Proc. 18th World Congress. The International Federation of Automatic Control. Milano (Italy) August 28 September 2, 2011.

[18]. D.Garcia, J.Luis,” Modeling and control of Squirrel Cage InductionGenerator with Full Power Converter applied towindmills," $\mathrm{PhD}$ thesis.

[19]. A. El magri, F. Giri , A. Elfadili " An Interconnected Observer for Wind Synchronous Generator,"in Proc. 8th IFAC Symposium on Nonlinear Control SystemsUniversity of Bologna, Italy, September 1-3, 2010.

[20]. Commande vectorielle sans capteur des machines asynchrones book of the author Claude Chaigne (5 août 2005). 